\title{
ON ESTIMATING THE HIGH-ENERGY CUTOFF IN THE X-RAY SPECTRA OF BLACK HOLES VIA REFLECTION SPECTROSCOPY
}

\author{
Javier A. García ${ }^{1}$, Thomas Dauser ${ }^{2}$, James F. Steiner ${ }^{1}$, Jeffrey E. McClintock ${ }^{1}$, Mason L. Keck ${ }^{1,3}$, and Jörn Wilms ${ }^{2}$ \\ ${ }^{1}$ Harvard-Smithsonian Center for Astrophysics, 60 Garden St., Cambridge, MA 02138 USA; javier@head.cfa.harvard.edu, \\ jem@cfa.harvard.edu, jsteiner@head.cfa.harvard.edu, keckm@bu.edu \\ ${ }^{2}$ Dr. Karl Remeis-Observatory and Erlangen Centre for Astroparticle Physics, Sternwartstr. 7, D-96049 Bamberg, Germany; \\ thomas.dauser@sternwarte.uni-erlangen.de \\ ${ }^{3}$ Institute for Astrophysical Research, Boston University, 725 Commonwealth Avenue, Boston, MA 02215, USA \\ Received 2015 March 1; accepted 2015 July 2; published 2015 July 27
}

\begin{abstract}
The fundamental parameters describing the coronal spectrum of an accreting black hole are the slope $\Gamma$ of the power-law continuum and the energy $E_{\text {cut }}$ at which it rolls over. Remarkably, this latter parameter can be accurately measured for values as high as $1 \mathrm{MeV}$ by modeling the spectrum of X-rays reflected from a black hole accretion disk at energies below $100 \mathrm{keV}$. This is possible because the details in the reflection spectrum, rich in fluorescent lines and other atomic features, are very sensitive to the spectral shape of the hardest coronal radiation illuminating the disk. We show that by fitting simultaneous $\operatorname{NuSTAR}(3-79 \mathrm{keV})$ and low-energy (e.g., Suzaku) data with the most recent version of our reflection model relxill one can obtain reasonable constraints on $E_{\text {cut }}$ at energies from tens of $\mathrm{keV}$ up to $1 \mathrm{MeV}$, for a source as faint as $1 \mathrm{mCrab}$ in a $100 \mathrm{ks}$ observation.
\end{abstract}

Key words: accretion, accretion disks - atomic processes - black hole physics

\section{INTRODUCTION}

$\mathrm{X}$-ray reflection spectroscopy is currently our most effective tool for probing accretion processes and spacetime near the supermassive black holes that power active galactic nuclei (AGNs). In the current paradigm, the power-law continuum is produced either in a central hot corona (e.g., Shakura \& Sunyaev 1973; Haardt 1993) or at the base of a jet (e.g., Matt et al. 1992; Markoff et al. 2005). A fraction of this radiation illuminates the accretion disk and is reprocessed into a rich reflection spectrum consisting of fluorescent lines and other features, which provides detailed information on the composition and ionization state of the accretion disk and constraints on the structure of the corona. Importantly, reflection spectroscopy of AGNs is the way to infer the spin of their black holes via modeling key spectral features, such as the $\mathrm{Fe} \mathrm{K}$ line $(\sim 6.4-6.9 \mathrm{keV})$, the Fe $\mathrm{K}$ edge $(\sim 7-8 \mathrm{keV})$, and the Compton hump $(\sim 20-40 \mathrm{keV})$. Observational data suggest that in many cases the illuminating power-law continuum cuts off abruptly in the hard X-ray band (e.g., Zdziarski et al. 2000). Most X-ray detectors are unable to accurately constrain this cutoff energy because they either have limited sensitivity at high-energies or none at all. Thus, reflection models have been traditionally simplified by adopting a fixed cutoff energy at a reasonable value, e.g., $E_{\text {cut }}=300 \mathrm{keV}$ (Ross \& Fabian 2005; García \& Kallman 2010).

The 2012 launch of NuSTAR (Harrison et al. 2010) has revolutionized reflection spectroscopy because its instruments provide both very low background and superb sensitivity over the band 3-79 keV, which captures all the key reflection features (e.g., Risaliti et al. 2013; Walton et al. 2013; Parker et al. 2014). The quality of the NuSTAR data demand reflection models that properly treat the high-energy cutoff as a fit parameter. We have developed advanced relativistic models of $\mathrm{X}$-ray reflection from ionized accretion disks; the most recent version is relxill(García et al. 2014). These models include a rich atomic database, full treatment of the angular distribution of the reflected radiation, and an improved geometrical model of the illuminating coronal source. Furthermore, the most recent version of relxill also includes the high-energy cutoff $E_{\text {cut }}$ as a fit parameter. Likewise, $E_{\text {cut }}$ is included as a free parameter in the most recent version of the reflection code reflionx (Ross \& Fabian 2005).

In the standard picture, the power law spectrum is generated in the corona by Compton up-scattering of thermal disk photons. The shape of the spectrum depends on the geometry, temperature, and optical depth of the corona (e.g., Rybicki \& Lightman 1979, p. 393). In particular, the fractional energy change of a scattered photon (in the non-relativistic limit)

$$
\frac{\Delta E}{E}=\frac{4 k T_{e}}{m_{e} c^{2}}-\frac{E}{m_{e} c^{2}}
$$

implies a cutoff of the power-law near $4 k T_{e}$ because the energy gained by the photon cannot exceed the energy of the electron (e.g., Titarchuk 1994). In practice, $E_{\text {cut }} \sim 2-3 k T_{e}$ due to dispersion, geometrical, and relativistic effects (e.g., Petrucci et al. 2001). Thus, an empirical estimate of $E_{\text {cut }}$ provides direct information on the temperature $T_{e}$ of the coronal electrons.

The recent data obtained with NUSTAR has spotlighted $E_{\text {cut }}$ (e.g., Ballantyne et al. 2014; Brenneman et al. 2014; Marinucci et al. 2014; Baloković et al. 2015). In most of these studies, the coverage of NUSTAR to $79 \mathrm{keV}$ was sufficient to directly observe the roll over of the power-law component $\left(E_{\text {cut }} \sim 50-200 \mathrm{keV}\right)$. However, fitting a NuSTAR spectrum of NGC 5506 with relativistic reflection models, Matt et al. (2015) surprisingly found $E_{\text {cut }}=720_{-190}^{+130} \mathrm{keV}$. Likewise, in fitting a Suzaku+NuSTAR spectrum of NGC 4151 Keck et al. (2015) found that a value of $E_{\text {cut }}$ near $1 \mathrm{MeV}$ was statistically required.

In this letter, we demonstrate the remarkable ability of reflection models to provide strong constraints on $E_{\text {cut }}$ up to several hundred $\mathrm{keV}$, values that greatly exceed the limit of the detector bandpass (e.g., $79 \mathrm{keV}$ for NUSTAR). While not detected directly, this high-energy portion of the spectrum conditions the ionization state and structure of the disk 
atmosphere, which in turn modifies the observed reflection features. Thus, by properly modeling the reflection component at moderate energies, one can accurately estimate $E_{\text {cut }}$ at extreme energies and thereby constrain the physical properties of the corona.

\section{MODELING THE REFLECTION SPECTRUM}

The reflection model relxill is a fusion of our reflection code xillver (García \& Kallman 2010; García et al. 2013) and relline (Dauser et al. 2010, 2013), which is a generalrelativistic ray tracing code. We first describe the atomic physics part xillver, which assumes plane-parallel geometry and idealizes the disk as a slab with a total optical depth of $\tau=10$ and a constant density of $n=10^{15} \mathrm{~cm}^{-3}$. The illuminating source is assumed to have a power-law spectrum with a photon index $\Gamma$ and an exponential cutoff at high energies, $E_{\text {cut }}{ }^{4}$ The intensity of the illumination is controlled by specifying the ionization parameter:

$$
\xi=\frac{4 \pi F_{x}}{n},
$$

where $F_{x}$ is the net ionizing flux in the energy band 1-1000 Ry. A comprehensive grid of models has been calculated and provided to the community for fitting observational data that cover a wide range of model parameters. The most recent version of xillverincludes models for several different values of the high-energy cutoff, namely, $E_{\text {cut }}=20,50$, $80,100,150,200,250,300,500,1000 \mathrm{keV}$. In Figure 1 we show examples of models for these values of $E_{\text {cut }}$ for two ionization parameters, $\log \xi=1$ and $\log \xi=3$.

As illustrated in Figure 1 and discussed in García et al. (2013), $E_{\text {cut }}$ controls the intensity and shape of the illuminating spectrum and thereby affects the global reflection spectrum. The Compton hump is most dramatically affected, its peak shifting to lower energies as $E_{\text {cut }}$ decreases. Weaker effects are seen at lower energies $(\lesssim 10 \mathrm{keV})$, which are mostly driven by changes in the ionization state of the gas. As Equation (2) suggests, two models with the same ionization parameter (and hence the same $F_{x}$ ) but different values of $E_{\text {cut }}$ will give rise to distinctive reflection spectra. This difference occurs because the illuminating spectrum with lower $E_{\text {cut }}$ will have a significantly larger proportion of its photons concentrated at lower energies, which favors photoelectric interactions over electron scattering relative to the spectrum with high $E_{\text {cut }}$.

Changes in $E_{\text {cut }}$ and the consequent shift in the balance of photons in the hard and soft bands affects the ionization structure of the slab, as illustrated in the two right panels of Figure 1. On the one hand, for low ionization the lower values of $E_{\text {cut }}$ (strong soft flux) produce hotter atmospheres; as a consequence, in this regime the temperature is mainly regulated by photoionization heating and recombination cooling. On the other hand, for high ionization the hotter atmospheres correspond to higher values of $E_{\text {cut }}$ (strong hard flux); in this regime, the temperature is controlled by electron scattering of the abundant hard photons. Finally, we stress that despite the opposing trends in the temperature profiles shown in Figure 1,

\footnotetext{
4 For simplicity, we use a phenomenological e-folded power-law model rather than a proper model of thermal Comptonization such as that of Zdziarski et al (2003), a model whose accuracy has been demonstrated (e.g., Gierlinski et al. 1997; Wardziński et al. 2002).
}

for a fixed value of $\xi$ the reflected spectrum is always softer for smaller $E_{\text {cut }}$.

To produce the complete relativistic model relxill, we convolve spectra computed along the disk using xillverwith the general-relativistic ray tracing code relline (Dauser et al. 2010, 2013). The model relxillself-consistently calculates the angle-dependent, relativistic ionized reflection spectra (García et al. 2014) that are used in fitting data. Figure 2 shows reflected spectra (and the incident power-law spectra) for $\log \xi=1$ calculated with relxill. For clarity, we show only two models, for $E_{\text {cut }}=$ $300 \mathrm{keV}$ and $E_{\text {cut }}=1 \mathrm{MeV}$. These are the same as the corresponding two models shown in the top-left panel of Figure 1, except that here the spectral features are blurred by Doppler and gravitational-redshift effects.

The lower panel in Figure 2 shows the ratio of the $E_{\text {cut }}=$ $300 \mathrm{keV}$ model to the $E_{\text {cut }}=1 \mathrm{MeV}$ model and also the ratio of the incident power-law spectra. In the NUSTAR band, while the two models can be distinguished by considering either the power-law or the reflected component, it is the stronger curvature of the reflected component that is more telling. At lower energies, in the Suzaku band, the power-law is incapable of distinguishing between the two models because the continuum is essentially identical (apart from the normalization). On the other hand, the reflected component shows significant changes in spectral features, which sharply distinguish the models. Thus, whether one considers NuSTAR alone, or NuSTAR plus Suzaku, it is possible to constrain $E_{\text {cut }}$ at energies far above the bandpass of the detector, but in either case it is the reflected component that carries the greater weight.

\section{FITS TO SIMULATED NUSTAR DATA}

We first demonstrate that $N U S T A R$ data alone can provide strong constraints on $E_{\text {cut }}$. To this end, we simulate data using appropriate response and background files, ${ }^{5}$ assuming a circular extraction region with $60^{\prime \prime}$ radius centered $1^{\prime}$ off axis. We combine the data from both NUSTAR telescopes (focal plane modules FPMA and FPMB) and simulate spectra for an exposure time of $100 \mathrm{ks}$.

We consider sources, both extragalactic and Galactic, with intensities/fluxes that cover the range normally encountered in practice. Using the IsIS package (Houck \& Denicola 2000), we grouped the data so that the minimum signal-to-noise ratio for each bin was 5 to ensure the soundness of our $\chi^{2}$ analysis. We additionally grouped the data to ensure appropriate sampling, given that the resolution of the NUSTAR detectors is $0.4 / 0.9 \mathrm{keV}$ at $6 / 60 \mathrm{keV}$. Specifically, we further binned the simulated data, which have uniform bin widths of $0.04 \mathrm{keV}$ over the entire energy band, to 0.2 and $0.4 \mathrm{keV}$ for energies below and above $20 \mathrm{keV}$, respectively. ${ }^{6}$

In the simulations, we used the model Tbabs*relxill (Section 2), where Tbabs models the interstellar absorption (Wilms et al. 2000). We simulated spectra for eleven values of the high-energy cutoff ranging from $50 \mathrm{keV}$ to $1 \mathrm{MeV}$. The other model parameters, which are the same for all simulations, are: the hydrogen column density $N_{\mathrm{H}}=5 \times 10^{21} \mathrm{~cm}^{-2}$;

\footnotetext{
5 http://www.nustar.caltech.edu/page/response_files

6 We found that binning the data to a few samples per resolution element is important: without this binning, the fitted values of $E_{\text {cut }}$ were consistently biased below the input. This result suggests more generally that fitting oversampled data is not good practice.
} 

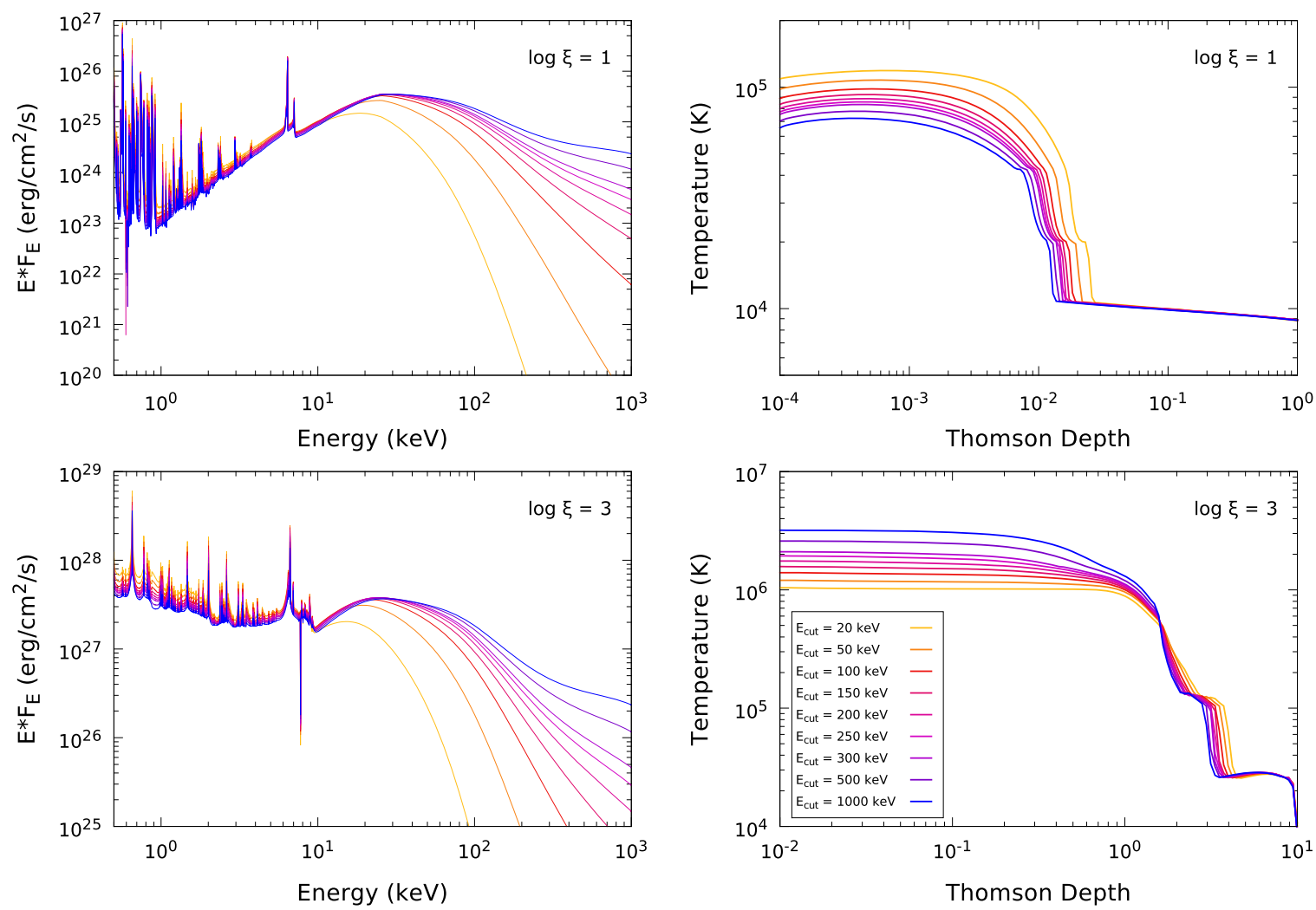

Figure 1. Left panels show xillver reflection spectra for $\log \xi=1$ (top) and $\log \xi=3$ (bottom) and for nine values of $E_{\text {cut }}$ (see legend in lower right panel). For all models, the illuminating spectrum is a power law with index $\Gamma=2$ and the iron abundance is solar. For simplicity we only show angle-averaged spectra (García et al. 2014). The right panels show the temperature profile in the slab.
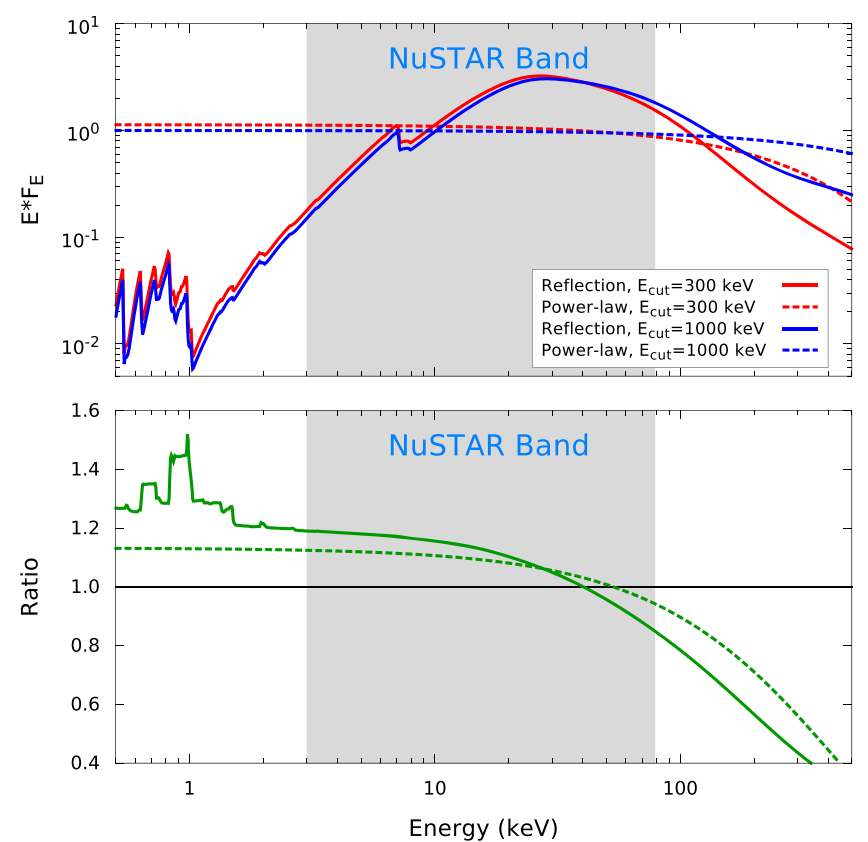

Figure 2. Top: relativistically blurred reflection spectra (solid lines) computed using relxill for $\log \xi=1$ and the respective power-law spectra (dashed lines) of the illuminating source with slope $\Gamma=2$. The red curves are for $E_{\text {cut }}$ $=300 \mathrm{keV}$ and the blue curves for $E_{\mathrm{cut}}=1 \mathrm{MeV}$. Bottom: ratio of the $E_{\text {cut }}=$ $300 \mathrm{keV}$ spectra to the $E_{\text {cut }}=1 \mathrm{MeV}$ spectra. The ratio of the reflection/ power-law spectra is plotted as a solid/dashed line. The shaded box spans the NuSTAR bandpass $(3-79 \mathrm{keV})$.

dimensionless spin parameter $a=c J / G M^{2}=0.998$; emissivity index $q=3$; inclination of the disk $i=45^{\circ}$; inner disk radius $R_{\text {in }}=R_{\mathrm{ISCO}}$; power-law $\Gamma=2$; ionization parameter $\log \xi=1$; iron abundance $A_{\mathrm{Fe}}=1$; and the reflection fraction $R_{f}=3$, which is defined as the ratio of the reflected to the power-law flux in the $20-40 \mathrm{keV}$ band. Our calculations in this instance do not link the reflection fraction to the emissivity index, which would require specifying the geometry of the reflector and the illuminating source.

We emphasize that this letter deals exclusively with relativistically blurred reflection by ionized gas in the very inner portion of the accretion disk for which the reflection fraction can vary widely, with values ranging from a few tenths to $>20$ for rapidly spinning black holes (Dauser et al. 2014). Reflection in this regime is not to be confused with reflection by distant, neutral material, which is observed in the spectra of many sources and is usually modeled using the pexrav model (e.g., Zdziarski et al. 1999, 2003).

We generated the simulated spectra using the fakeit task in XSPEC v-12.8.2b (Arnaud 1996), and we then fitted these spectra using two different models, with $E_{\mathrm{cut}}, \Gamma, \xi, A_{\mathrm{Fe}}$, normalization, and $R_{f}$ free to vary:

Model 1: Tbabs*relxill. We first employ the same model used to produce the simulated data in order to evaluate how well the parameters, $E_{\text {cut }}$ in particular, can be recovered.

Model 2: Tbabs*highecut*relxill. In this case, the parameter $E_{\text {cut }}$ in relxill is fixed at $1 \mathrm{MeV}$, while it is allowed to vary freely in highecut, a simple exponential convolution model acting on relxill. Model 2 aims to test how accurately $E_{\text {cut }}$ is recovered when the spectrum is described by modifying the high-energy region of an otherwise incorrect reflection model (i.e., when ignoring the effects on the disk's ionization structure described in Section 2).

Results of fitting the simulated data are shown in Figure 3. The top panels show the fitted values of $E_{\text {cut }}$ versus the values 

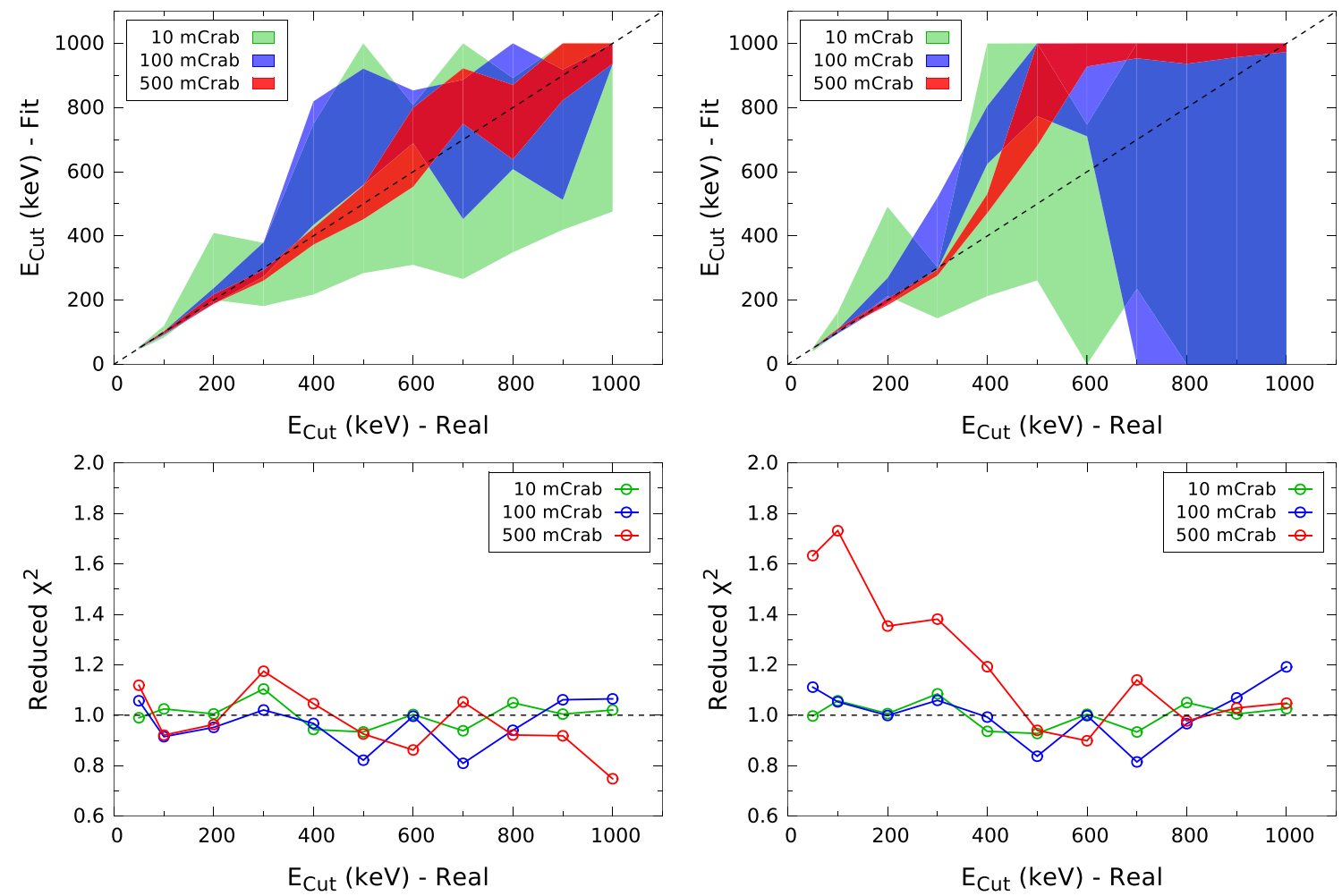

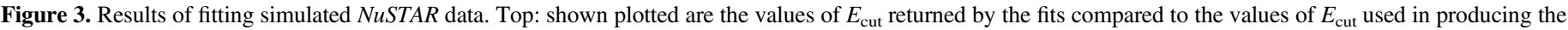

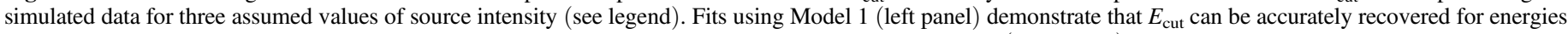

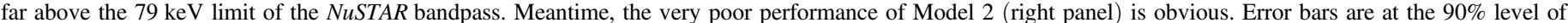
confidence. Bottom: goodness of fit; note the poor performance of Model 2 at low to moderate values of $E_{\text {cut }}$ for the brightest source.

used in simulating the data, with results for Model $1 / 2$ in the left/right panel. The shaded regions indicate uncertainties at the $90 \%$ confidence level. The colors are keyed to source intensity. The quality of the fits is indicated in the lower panels.

Focusing on the results for Model 1 (top-left panel) and the $10 \mathrm{mCrab}$ source, which delivers $1.8 \times 10^{6}$ counts in the $N U S T A R$ band, the recovered values of $E_{\text {cut }}$ below $400 \mathrm{keV}$ are of marginal quality, while above that energy they only provide lower limits on $E_{\text {cut }}$. However, for source intensities of $\sim 100-500 \mathrm{mCrab}\left(\sim 10^{7}-10^{8}\right.$ counts $)$, significantly better performance is achieved. We conclude that for high-quality data one can determine the high-energy cutoff with reasonable precision using $N U S T A R$ data alone.

Turning to the results for Model 2 (top-right panel), one immediately sees the much poorer performance achieved in attempting to recover $E_{\text {cut }}$ by simply fitting the curvature in the power-law continuum component using the highecut model. As mentioned in Section 1, this was the standard approach used before $E_{\text {cut }}$ was included as a fit parameter in reflection models. The highecut model provides constraints of reasonable quality only for $E_{\text {cut }}<300 \mathrm{keV}$. Furthermore, for the brightest source considered ( $500 \mathrm{mCrab})$, Model 2 yields unsatisfactory results for $E_{\text {cut }}>300 \mathrm{keV}$ because not only is $E_{\text {cut }}$ significantly over-predicted, the error bars are also misleadingly small. Interestingly, at lower energies the quality of the fits are worse for the brightest source (Figure 3, bottom-right panel). Other model parameters are also poorly constrained, e.g., the Fe abundance is grossly underestimated, while both the power-law index and the reflection fraction $R_{f}$ are over-predicted.

Importantly, we note that Models 1 and 2 give the same quality of fit (with the exception of the 500 mCrab simulation for low $\left.E_{\text {cut }}\right)$ so that a $\chi^{2}$-test does not generally promote one model over the other. However, as we have demonstrated, Model 2 returns incorrect estimates of the parameters in all cases. In short, good statistical precision does not ensure that the parameter values returned by a fit to a model are reliable or physically meaningful.

\section{INCLUDING LOW-ENERGY DATA}

Figure 2 indicates that the low-energy component $(E \lesssim 3$ $\mathrm{keV}$ ) of the reflection spectrum is more sensitive to the value of $E_{\text {cut }}$ than the component in the NuSTAR band. Therefore, we now consider spectra comprised of Suzaku plus NuSTAR data in the expectation that this will significantly improve the constraints on $E_{\text {cut }}$, and the other parameters as well. The simulated low-energy data are prepared using Suzaku response files, the same set of model parameters, and the same source fluxes as those used in preparing the NUSTAR data.

In the following, we consider only Model 1. Fitting the combined set of Suzaku $(0.5-8 \mathrm{keV})$ and NuSTAR $(3-79 \mathrm{keV})$ simulated spectra, we obtain the outstanding result shown in Figure 4. The fit for the $10 \mathrm{mCrab}$ source is incomparably better than for the NUSTAR data alone (compare upper left panel of Figure 3). The high-energy cutoff is strongly constrained for all values of $E_{\text {cut }}$ considered; even at $1 \mathrm{MeV}$, the error is only about $7 \%$. This excellent performance of our reflection model is a consequence of the sensitivity of the reflection component to changes in the high-energy portion of the illuminating spectrum (Figure 2). These simulations clearly demonstrate that fits to features in the relatively low-energy portion of the spectrum, where the instruments are most 


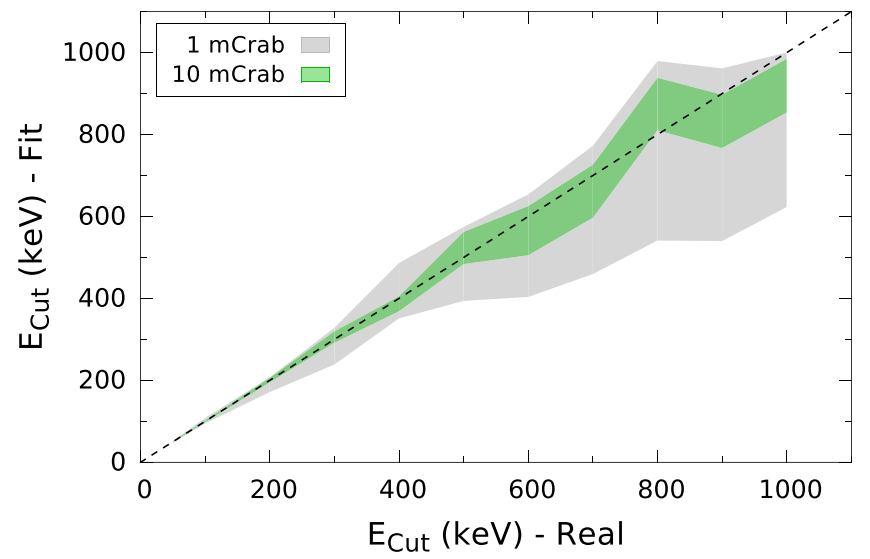

Figure 4. Including low-energy Suzaku data, Model 1 delivers extraordinary performance in constraining $E_{\text {cut }}$ out to extreme energies compared to using $N u S T A R$ data alone. The results shown here of fitting simulated data for a $10 \mathrm{mCrab}$ source are superior to those obtained for a $500 \mathrm{mCrab}$ source using only $N u S T A R$ data (see the upper left panel in Figure 3).

sensitive, can deliver accurate measurements of $E_{\text {cut }}$ at energies far beyond the limit of the detector bandpass.

Also shown in Figure 4 are the results for a $1 \mathrm{mCrab}$ source $\left(2 \times 10^{5}\right.$ counts $)$. Remarkably, with the inclusion of low-energy data even $\sim 10^{5}$ counts are sufficient to recover $E_{\text {cut }}$ with reasonable precision; the error bars for $E_{\text {cut }}>600 \mathrm{keV}$ are $\sim 30 \%$.

Finally, with two additional simulations we demonstrate that the reflection component is much more important in constraining $E_{\text {cut }}$ than the power law. For the same $1 \mathrm{mCrab}$ source, and including Suzaku data, Figure 5 compares the performance of a cutoff power-law model alone (cutoffpl) with a pure reflection model. These two limiting cases can be compared directly to the intermediate case of our adopted composite model (i.e., power law plus reflection) with $R_{f}=3$ shown in gray in Figure 4 . The three cases considered together show that stronger reflection translates into a better constraint on $E_{\text {cut }}$.

\section{DISCUSSION AND CONCLUSIONS}

We have demonstrated that our reflection model relxill can constrain the physically important coronal parameter $E_{\text {cut }}$ for spectra with intensities like those encountered in observations of AGNs. We have furthermore shown that attempting to constrain $E_{\text {cut }}$ by imposing a cutoff that is external to the reflection model yields relatively poor constraints on $E_{\text {cut }}$. Such a simplified model inaccurately represents the observed spectrum and introduces strong biases in the values inferred for the key physical parameters.

We analyzed two simulated data sets using relxill: (1) With NuSTAR data alone (3-79 keV), $E_{\text {cut }} \gtrsim 400 \mathrm{keV}$ can only be constrained if the spectrum contains $\gtrsim 10^{7}$ counts, which is infeasible for most observations of AGNs. (2) However, our simulations indicate that for a simultaneous fit to Suzaku and NuSTAR data $(0.5-79 \mathrm{keV})$, one can constrain $E_{\text {cut }}$ for a $1 \mathrm{mCrab}$ source at energies far above the limit of the detector bandpass.

Our results lead to two basic conclusions.

1. In constraining the cutoff energy, misleading results are obtained if the power-law component is cut off externally. It is therefore essential to employ a detailed

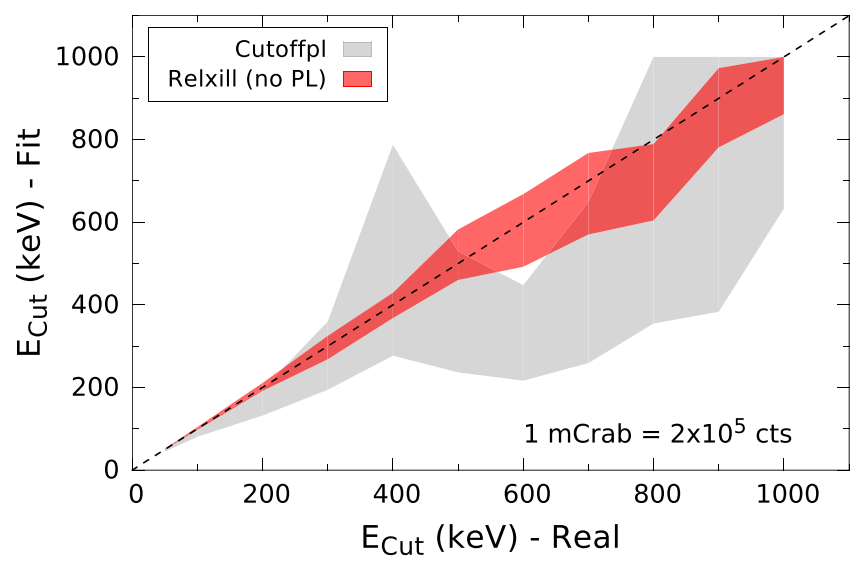

Figure 5. Results simulated for a $1 \mathrm{mCrab}$ source demonstrating that a pure reflection model (i.e., no power-law continuum) shown in red is far superior to an e-folded power-law component (i.e., no reflection) in constraining $E_{\text {cut }}$.

and accurate model of the reflection spectrum that includes $E_{\text {cut }}$ as an internal fit parameter.

2. Because the cutoff energy most strongly affects the lowenergy part of the reflection spectrum, to obtain useful constraints on $E_{\text {cut }}$ for AGNs it is crucial to combine NuSTAR data with low-energy (e.g., Suzaku or XMMNewton) data.

This limited study provides only a very cursory exploration of how proper modeling of a reflection spectrum yields useful physical constraints on the high-energy cutoff out to extreme energies. A thorough exploration of the subject is well beyond the scope of this letter. For example, we illustrate the effects on the reflected spectrum of varying $E_{\text {cut }}$ for the cases of $\log \xi=1$ and 3 (Figure 1) while only presenting simulations for the former case. However, one expects that the effects will be quite different for other choices of the ionization parameter. Moreover, the values of other parameters, such as the reflection fraction, can affect the statistical significance of the value returned for $E_{\text {cut }}$.

J.G. and J.E.M. acknowledge the support of NASA grant NNX11AD08G. J.F.S. has been supported by NASA Hubble Fellowship HST-HF-51315.01.

\section{REFERENCES}

Arnaud, K. A. 1996, in ASP Conf. Ser. 101, Astronomical Data Analysis Software and Systems V, ed. G. H. Jacoby \& J. Barnes (San Francisco, CA: ASP), 17

Ballantyne, D. R., Bollenbacher, J. M., Brenneman, L. W., et al. 2014, ApJ, 794, 62

Baloković, M., Matt, G., Harrison, F. A., et al. 2015, ApJ, 800, 62

Brenneman, L. W., Madejski, G., Fuerst, F., et al. 2014, ApJ, 788, 61

Dauser, T., García, J., Parker, M. L., Fabian, A. C., \& Wilms, J. 2014 MNRAS, 444, L100

Dauser, T., Garcia, J., Wilms, J., et al. 2013, MNRAS, 430, 1694

Dauser, T., Wilms, J., Reynolds, C. S., \& Brenneman, L. W. 2010, MNRAS, 409, 1534

García, J., Dauser, T., Lohfink, A., et al. 2014, ApJ, 782, 76

García, J., Dauser, T., Reynolds, C. S., et al. 2013, ApJ, 768, 146

García, J., \& Kallman, T. R. 2010, ApJ, 718, 695

Gierlinski, M., Zdziarski, A. A., Done, C., et al. 1997, MNRAS, 288, 958

Haardt, F. 1993, ApJ, 413, 680

Harrison, F., Boggs, S., Christensen, F., et al. 2010, BAAS, 41, 737

Houck, J. C., \& Denicola, L. A. 2000, in ASP Conf. Ser. 216, Astronomical Data Analysis Software and Systems IX, ed. N. Manset, C. Veillet, \& D. Crabtree (San Francisco, CA: ASP), 591 
Keck, M. L., Brenneman, L. W., Ballantyne, D. R., et al. 2015, ApJ, 806, 149 Marinucci, A., Matt, G., Kara, E., et al. 2014, MNRAS, 440, 2347

Markoff, S., Nowak, M. A., \& Wilms, J. 2005, ApJ, 635, 1203

Matt, G., Baloković, M., Marinucci, A., et al. 2015, MNRAS, 447, 3029

Matt, G., Perola, G. C., Piro, L., \& Stella, L. 1992, A\&A, 257, 63

Parker, M. L., Wilkins, D. R., Fabian, A. C., et al. 2014, MNRAS, 443, 1723

Petrucci, P. O., Haardt, F., Maraschi, L., et al. 2001, ApJ, 556, 716

Risaliti, G., Harrison, F. A., Madsen, K. K., et al. 2013, Natur, 494, 449

Ross, R. R., \& Fabian, A. C. 2005, MNRAS, 358, 211

Rybicki, G. B., \& Lightman, A. P. 1979, Radiative Processes in Astrophysics (New York: Wiley-Interscience)
Shakura, N. I., \& Sunyaev, R. A. 1973, A\&A, 24, 337

Titarchuk, L. 1994, ApJ, 434, 570

Walton, D. J., Nardini, E., Fabian, A. C., Gallo, L. C., \& Reis, R. C. 2013, MNRAS, 428, 2901

Wardziński, G., Zdziarski, A. A., Gierliński, M., et al. 2002, MNRAS, 337,829

Wilms, J., Allen, A., \& McCray, R. 2000, ApJ, 542, 914

Zdziarski, A. A., Lubiński, P., Gilfanov, M., \& Revnivtsev, M. 2003, MNRAS, 342,355

Zdziarski, A. A., Lubiński, P., \& Smith, D. A. 1999, MNRAS, 303, L11

Zdziarski, A. A., Poutanen, J., \& Johnson, W. N. 2000, ApJ, 542, 703 